\title{
An array effect of wave energy farm buoys
}

\author{
Hyuck-Min Kweon ${ }^{1}$ and Jung-Lyul Lee ${ }^{2}$ \\ ${ }^{I}$ Department of Railroad, Construction and Environment System Engineering, Gyeongju University, Korea \\ ${ }^{2}$ Department of Civil and Environmental Engineering, Sungkyunkwan University, Suwon, Korea
}

\begin{abstract}
An ocean buoy energy farm is considered for Green energy generation and delivery to small towns along the Korean coast. The present studypresents that the floating buoy-type energy farm appears to be sufficiently feasible fortrapping more energy compared to afixed cylinder duck array. It is also seen from the numerical resultsthat the resonated waves between spaced buoys are further trapped by floating buoy motion. Our numerical study is analyzed by a plane-wave approximation, in which evanescent mode effects are included in a modified mild-slope equation based on the scattering characteristics for a single buoy.
\end{abstract}

KEY WORDS: Ocean buoy; Energy farm; Wave resonance; Wave-structure interaction.

\section{INTRODUCTION}

The oceans have been long taken into account as a great potential source of clean energy, even though other sources of renewable energy, such as wind and solar, have been widely adopted in recent years. Ocean waves carry tremendous power, and so wave converters could provide a major contribution to mitigating carbon dioxide emissions. European Thematic Network on Wave Energy (ETNWE, 2003) estimated that the worldwide amount of economically recoverable wave energy is in the range of 140 to $750 T W h / y r$ for existing fully mature wave energy converting technologies. This could be increased by a factor of 2 to 3 with projected long-term technical improvements (Thorpe, 1999).

Various types of energy converting technologies have been proposed to capture the energy of ocean waves in near shore, offshore, and far offshore locations, and they are installed at or near the water's surface, with which they usually interact to convert the energy of the waves into other energy forms, such as electricity. Some of the more promising design locations have been demonstrated as technically feasible and are undergoing feasibility testing in the field at commercial scales.

In the present study, we consider a wave energy farm technique for harnessing the wave energy produced by resonant heaving buoys and converting it to electricity. The present buoy system heaves with ocean waves, and the dimensions of the buoy are determined so as to help heaving motion in the frequency range of the most frequent incidence in ocean waves.

The wave energy farm has strong potential as a worldwide supplier of natural energy, producing no pollutants or chemical bi-products. The world's first commercial wave farm is the Aguçadora Wave Park located in Portugal near Póvoa de Varzim, which began with three Pelamis machines capable of generating a total of $2.25 \mathrm{MW}$. There are plans to increase this capacity to $21 \mathrm{MW}$ using an additional 25 Pelamis machines. Korea has no commercial wave farms, but construction plans have begun off the Donghae coast for the first wave-energy farm of ocean buoys to supply power to coastal towns.

Wave energy conversion schemes are generally anticipated to cause environmental changes with respect to marine habitat, sediment transport, toxic releases, and conflict with other sea space users. The impacts that could potentially occur are also very site-specific, depending on physical layout and conversion technologies. However, wave energy farms can be considered as al-

Corresponding author: Jung-Lyul Lee

e-mail: jllee@skku.edu 
ternative tools to control wave heights to an acceptable level in order to solve various problems associated with hard coastal structures (Lee et al., 2011). Furthermore, they are also favored in terms of coastal environments, since they do not generally partition the natural sea.

In this study, we accomplish a feasibility analysis of the generation potential off the eastern coast of Korea and present a numerical scheme to examine the effect of buoy arrays in wave energy generation. Our numerical study is accomplished by a plane-wave approximation in which both radiating and scattering waves due to floating devices are solved using a plane wave method without the inclusion of evanescent modes.

\section{A FEASIBILITY STUDY}

The eastern coast of Korea is interested in developing a wave energy farm to harvest wave energy as a clean, renewable resource. The generating potential of a given site is estimated by the power fluctuation of a wave. To have commercial interest, an annual average wave power fluctuation of about 20 to 50 kilowatts per meter is required in deep water.

The generating potential of the eastern coast was estimated based on wave data measured off the Hujung coast (Fig. 1(a)). Fig. 1 shows the average monthly significant wave height (in meters), period (in sec), and energy power flux (in $K W / m e t e r$ ).

The annual average wave power is not sufficient for commercial interest. So, we are devising a resonant buoy type and further designing its optimal array to amplify the generating potential so as to reach a commercial level. When a heaving function is applied for a floating buoy with a full length of $9 \mathrm{~m}$ and diameter of $3 \mathrm{~m}$, as shown in Fig. 2(a), commercial-level wave power amplification is obtained, as shown in Fig. 2(b).

The result is now comparable with the wave power off the Oregon coast, which is purely calculated from long term wave data over the past 10 years. The Oregon coast has been well known to have one of the richest ocean wave energy potentials in the world.

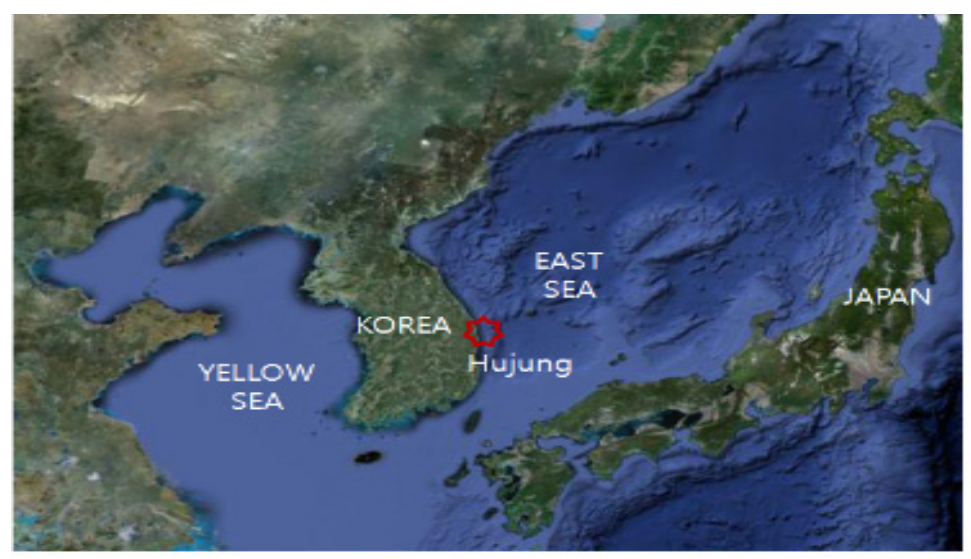

(a)

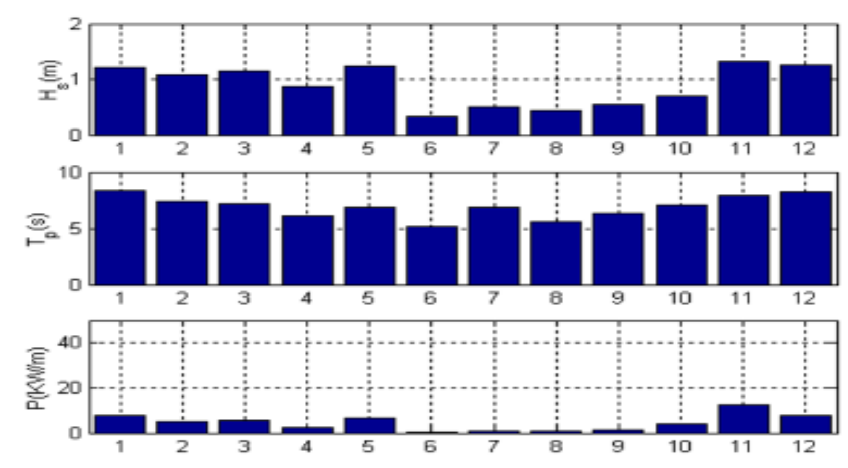

(b)

Fig. 1 (a) Hujung location and (b) wave power calculated from Hujung significant wave data. 


\section{WAVE SCATTERING DUE TO FLOATING BUOYS}

\section{Plane wave approach}

In an energy farm region composed of multiple floating structures, incoming waves may be significantly diffracted and scattered. Multiple bodies can be studied with the same theoretical and numerical methods that are applied to waveeffects on a single body (McIver and Evans, 1984; Simon, 1982; Mavrakos and McIver, 1997; Williams and Li, 2000). Typically, the analysis of two or three interacting bodies is a straightforward extension, but the analysis of very large configurations is fundamentally more difficult. Thus, there is a great need for a reliable numerical model suitable for analyzing the effect of wave scattering between buoys.

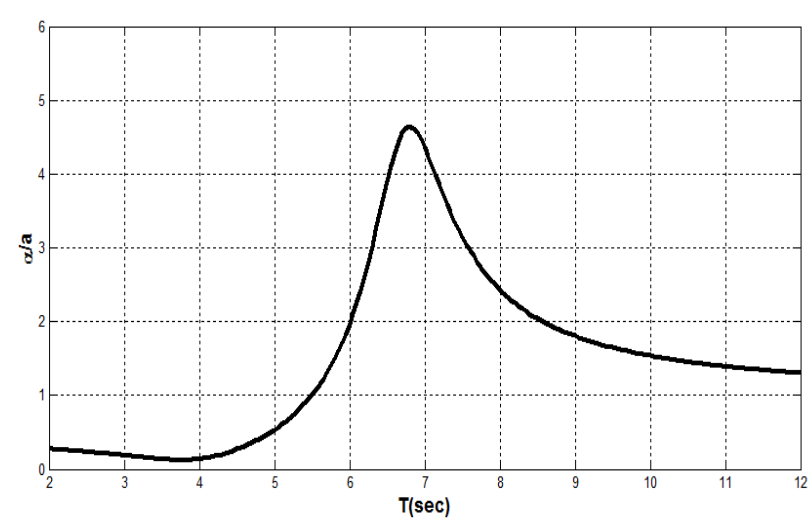

(a)
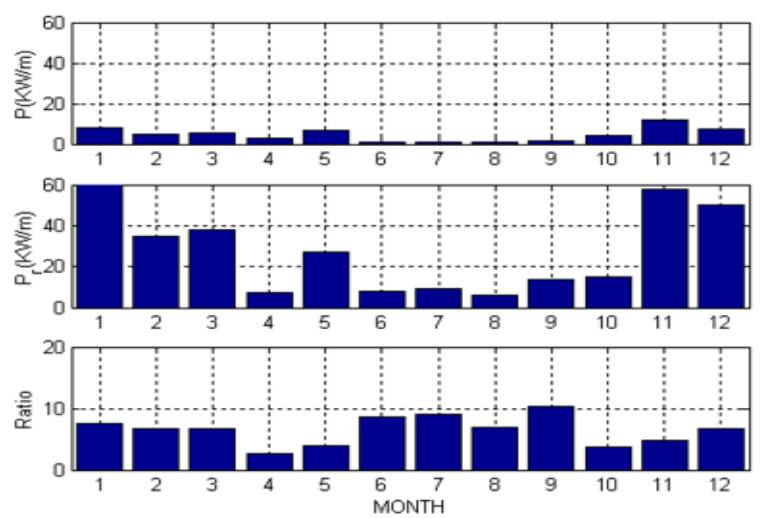

(b)

Fig. 2 (a) Variation of buoy resonant height with respect to wave period (Berteaux,1976) and

(b) wave power amplification ratio by buoy resonant effect.

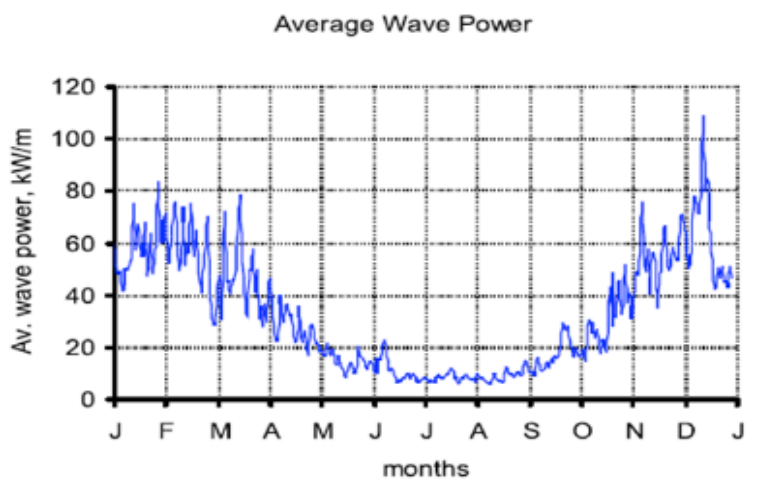

Fig. 3 Monthly average wave power flux at Oregon coast (Oregon Sea Grant Report, 2004).

The interaction between water waves and arrays of bodies has become increasingly important and remarkable numerical approaches of practical use have been presentedin recent years. Sawaragi et al. (1989) proposed a numerical method combining a three-dimensional.

Green's function model for near-field waves around a floating body and a two-dimensional BIEM model for far-field waves. Ohyama and Tsuchida (1997) expanded the mild-slope equation, incorporating evanescent modes to obtain significantly higher computational efficiency compared to previous approaches. However, their equation set for evanescent modes still requires significant CPU time and computational storage.

As a simpler approach, Lee and Lee (2001) and Lee and Lee (2003) proposed a plane wave approximate method to add scattering source terms to the traditional Mild-Slope Equation (MSE) without the inclusion of evanescent modes. The scattering term, which generates the scattering waves, is determined by the gradient of surface velocity potential across the breakwater. Evanescent modes are only considered in predicting the reflection ratio against the vertical breakwater. 
The MSE developed by Berkhoff (1972) has not only been used in its original form of an elliptic equation but has also provided a basic governing equation for the development of other wave equations such as the parabolic equation (Radder, 1979) and the hyperbolic equation (Smith and Sprinks, 1975; Copeland, 1985; Madsen and Larsen, 1987). Models based on this equation have been widely used in determining the comprehensive motion of linear monochromatic waves in areas of varying water depths. Recently, this equation was modified for several purposes, such as applicability to a steep bed profile, wave-structure interactions, and nonlinear wave propagation.

\section{Governing equation of MSE type}

Similarly to Madsen and Larsen's method(1987) for regular waves, the mild slope equations of shallow water equation type are reformulated by extracting the harmonic time variation while letting $\eta=S \exp (i \sigma t)$ and $\mathbf{u}=\mathbf{U} \exp (i \sigma t)$ in order to considerably speed up the solution since the wave period no longer needs to be resolved.

$$
\begin{gathered}
\frac{\partial^{2} S}{\partial t^{2}}+2 i \sigma \frac{\partial S}{\partial t}+\nabla \cdot\left[\frac{C C_{g}}{g}\left(\frac{\partial \mathbf{U}}{\partial t}+i \sigma \mathbf{U}\right)\right]-k^{2} C C_{g} S=S_{i} \\
\frac{\partial^{2} \mathbf{U}}{\partial t}+i \sigma \mathbf{U}+g \nabla S=\mathbf{U}_{s}
\end{gathered}
$$

where, $C$ is the wave celerity, $C_{g}$ is the group velocity, $g$ is the gravitational acceleration, $S_{i}$ is the wave generating source term which generates the incoming waves and $\mathbf{U}_{s}$ is the scattering source term. The wave generating source term is given in terms of the incident wave height $H_{i}$ on the grid mesh of $\Delta x$ and $\Delta y$ :

$$
S_{i}=\sigma C_{g} H_{i} \exp \left(i k \sin \theta_{i} y\right) \frac{\Delta s}{\Delta x \Delta y}
$$

where $\theta_{i}$ is the incident wave direction, and $\Delta s$ is the width of the incident wave front inside a mesh. A new set of combined differential equations, (1a) and (1b), as shown above, can be applied in most effective engineering practices to assess the iregular wave conditions as well as regular wave conditions in existing or proposed new harbors.

A floating device can be taken into account as another source because it generates scattering waves. As shown in Eq. (1a), the scattering term is given in the momentum equation since it is expressed in terms of $\mathbf{U}_{s}$ as

$$
\mathbf{U}_{s}=2 C \frac{r}{t} \frac{\Delta s}{\Delta x \Delta y} \mathbf{U}
$$

where $\Delta s$ is the occupied width of the device inside a mesh and $t$ and $r$ are the transmission and reflection coefficients of a device element, respectively. The more general description of $r / t$ will be shown later in terms of wave reflection, transmission and energy absorption in this section. In this plane wave approach, only the evanescent mode effects are considered in predicting the ratio $r / t$ against a heaving structure.

Hereafter, the minus sign is removed from the signs after a comparison with the well-known single-barrier solutions of Ursell and Dean (1947). The conventional mild-slope equation was modified with an additional term inserted.

The governing equations are solved by the approximate factorization techniques leading to the implicit finite difference schemes. The scheme is applied with the complex variables defined on a space-staggered rectangular grid. Since the matrix coefficients of the corresponding difference equations form a tri-diagonal matrix, they are solved using the tri-diagonal algorithm which is widely used in solving difference equations because of its great efficiency. A more detailed description including the treatment of side boundary conditions and partial reflecting wall conditions is given by Lee and Lee (2001). MSE model 
results werecompared and were in good agreement with the mathematical results for two and three identical barriers of equal length (Kweon et al., 2011).

\section{Wave absorbing of a single buoy}

Once the device motion is estimated, the efficiency of wave absorption can be obtained and the values are applied to nearfield waves. This process should be repeated until the steady state solution is maintained. In the present study, however, the constant value is used for the test of model performance. The power per unit length in a sinusoidal two-dimensional progressive wave is the mean energy flux per unit length crossing a vertical plane normal to the direction of the wave. For the efficiency of a heaving device $e$, we obtain the following in terms of reflection and transmission coefficients (Evans, 1976):

$$
r_{-} t_{+}^{*}+r_{+}^{*} t_{-}=e
$$

where $(*)$ denotes the complex conjugate. Multiplying Eq. 4 by $t_{+} r_{+}$and dividing by $t_{-} t_{+}$yields:

$$
\left|t_{+}\right|^{2} \frac{r_{-} r_{+}}{t_{-} t_{+}}-e \frac{r_{+}}{t_{-}}+\left|r_{+}\right|^{2}=0
$$

where $t_{-}=t_{+}=t$. In the case of a symmetric body, $r_{-}=r_{+}=r$. Thus Eq. (5) becomes

$$
\left|t_{+}\right|^{2} \frac{r^{2}}{t^{2}}-e \frac{r}{t}+\left|r_{+}\right|^{2}=0
$$

which yields the solutions:

$$
\left.\alpha=\frac{r}{t}=\frac{e / 2-\sqrt{e^{2} / 4-|r|^{2}|t|^{2}}}{|t|^{2}}\right]
$$

This result is used with Eq. (3). In the present simulation, the device occupies more than one grid. Thus, the transmission, reflection, and absorption coefficients, $|t|,|r|$ and $e$, can vary according to the grid position, and they are determined by comparing with analytical solutions of surface velocity potential or measured values of surface level obtained along the boundary of a single device.

\section{WAVE SCATTERING DUE TO FLOATING BUOYS}

\section{Buoy arrays}

Twelve cylinders of equal diameter as shown in Fig. 4 are considered to compare the wave extracting efficiency for the respective arrays of circular and linear types with the distance shown in Fig. 4(a) and 4(b). The computational domain is composed of a mesh of $200 \times 200$ grid squares with a grid spacing of $0.5 \mathrm{~m}$ and the diameter of the floating cylinder is $3.5 \mathrm{~m}$. Thirty seven grids are occupied by a cylinder as shown in Fig. 4(c). Numerical results for both fixed dock and floating buoy cases are obtained at a uniform water depth of $30 \mathrm{~m}$.

In the present study, the array effect is examined for the short wave conditions ( $T=3 \sim 6 \mathrm{sec}$.) out of a heaving period zone, and uniform mild wave conditions $\left(H_{i}=0.5 \mathrm{~m}\right)$. The model was run for 2 different incident angles of 0 and $45^{\circ}$, and about 18 different wave periods from 3 to $6 \mathrm{sec}$ to find an optimal period. 
Each floating buoy is assumed to transmit $40 \%$ of the energy since the transmissioncoefficient reaches the values of about 0.4 under the forcing of short period waves inintermediate and deep waters (Koutandos et al., 2005). Evans (1976) showed that the maximum efficiency of wave absorption for a rolling vertical cylinder reaches $50 \%$ because of the horizontal symmetry about the axis of oscillation. In the present study, we adopt $25 \%$ as one half of full efficiency.

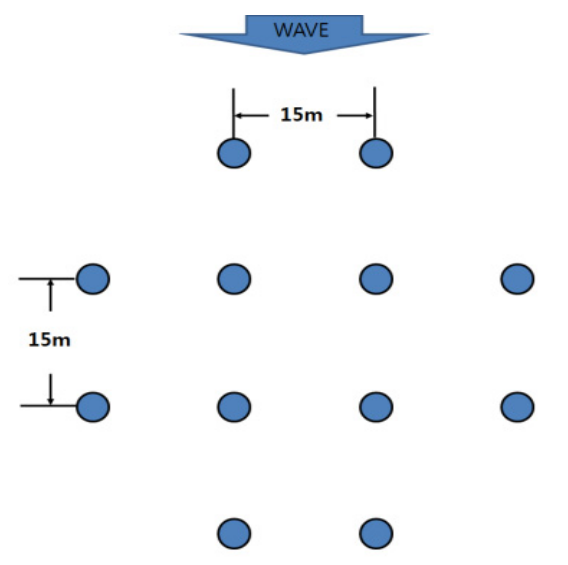

(a)

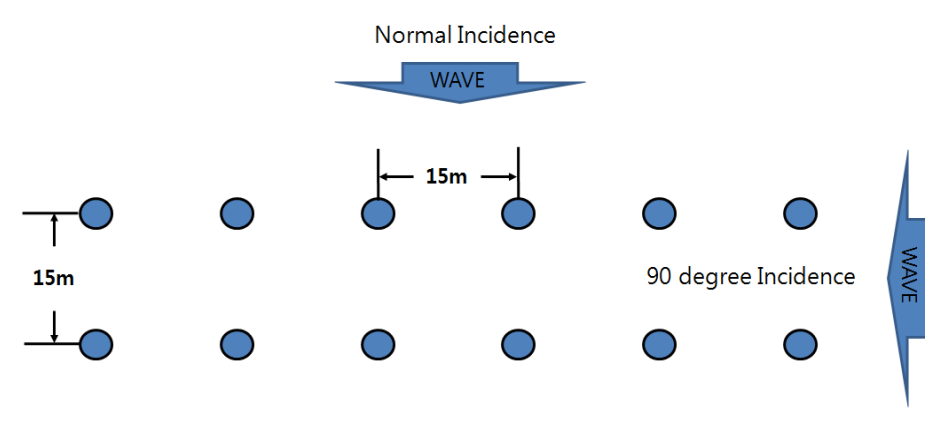

(b)

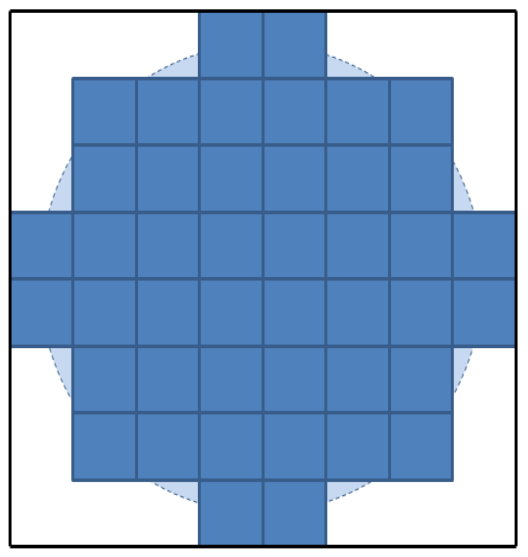

(c)

Fig. 4 (a) Circular array, (b) linear array of two rows and (c) grid assignment of a cylinder.

\section{Numerical results}

A numerical experiment has been conducted in order to investigate the mechanism of the wave energy magnification effect caused by the wave energy of buoy groups. The results are compared between numerical experiments, as shown in Fig. $5 \sim 9$. The wave energies are measured along the duck-bounded grids for fixed cylindrical duck cases and at grids where buoys are arranged for floating cases.

Fig. 5 shows the wave energy magnification factors of both array types for the fixed cylindrical duck casesvarying with respect to incident wave periods. The factor of circular array shows more variation with several peaks when compared with those of the linear array (normal incidence as shown in Fig. 4(b)), and indicates more wave energy trapping near a wave period of 4 sec. $(k=0.25)$, and less trapping near $T=3.1(k=0.42)$ and 4.5 seconds $(k=0.20)$. The maximum energy magnification factor reaches a value of 1.6 by a circular array in the given period interval.

Fig. 6 shows the factorvariation of both array types for the floating buoy cases. An interesting feature of floating buoy array problems is the smoothness in the variation of energy magnification factors with respect to wave periods. It is also interesting that the floating buoy results, as shown in Fig. 7, indicate more energy magnification effect than the fixed, although they may be expected to cause more wave energy trapping due to perfect reflection of a single duck. 
Judging from the array feature, the circular array may not be verysensitive to incident angles, but the linear one may show significant change inthemagnification factor by incident angles. Therefore, further numerical computation was conducted in order to investigate the incident angle effect for a linear array. As shown in Fig. 8, the results for a right angle (90 degree) incidence show prominent increases in the factor when compared with the others, except for a sudden decrease in the neighborhood of $T=4.5$ seconds.

We now examine the effect of buoy diameter on an energy magnification factor. As shown in Fig. 9, the twice-bigger diameter contributes a significant increase in the factor in the range of $T=3.5 \sim 4.5$ seconds. The peak periods seem to be related to not only buoy spacing but also diameter size, which may indicate that the resonated waves between spaced buoysare further trappedbyfloating buoy motion.

Fig. 10 shows the wave height distributions for floating buoy-type arrays for $T=3.5$ seconds .in which a high magnification factor is obtained, while Fig. 11 shows the results for $T=4.5$ seconds in which a low magnification factor is obtained. Buoy positions are indicated by dotted white circles in the figures.

It is clearly seen that for $T=3.5 \mathrm{sec}$. the energy farm absorbs more wave energy, thus reducing the wave reflection against the farm, for $T=4.5 \mathrm{sec}$. the energy farm significantly reflects the incident wave energies. Of particular interest in the results is the wave energy trapping showing high wave heights depicted at the position where floating buoys are located.

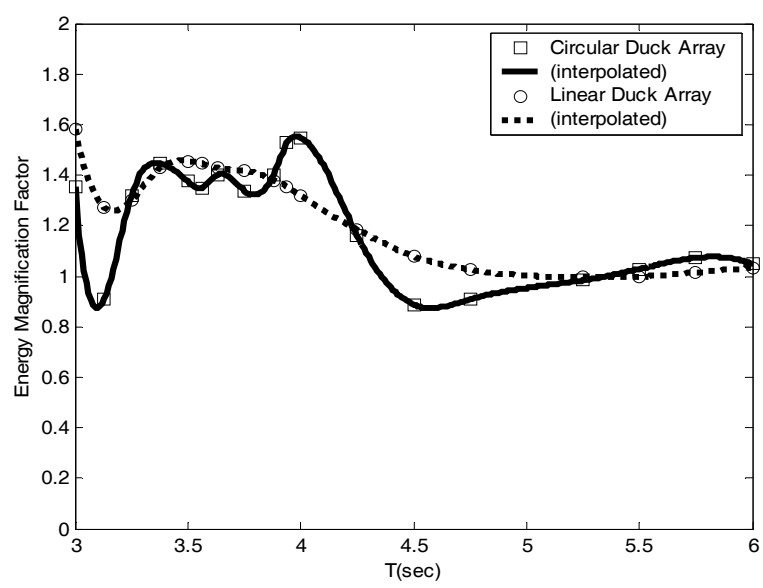

Fig. 5 Comparison between array types for energy magnification factor measured at cylinder duck faces.

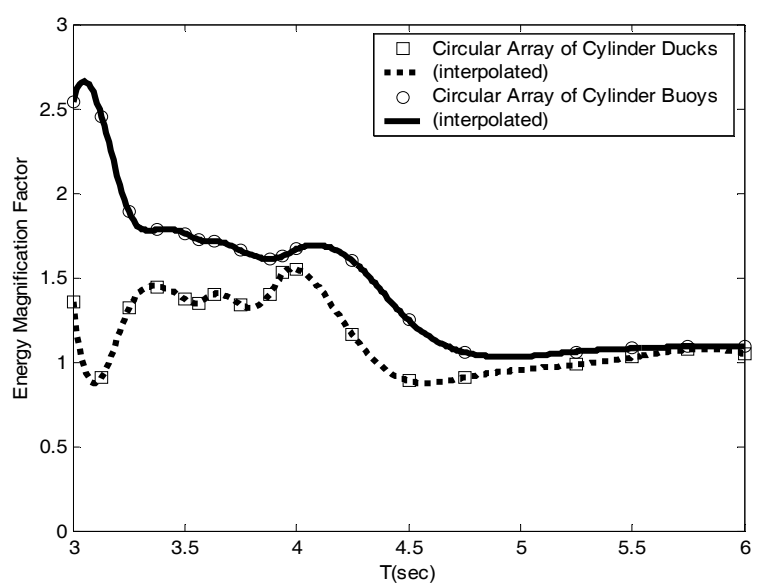

Fig. 7 Comparison of energy magnification factors between fixed duck and floating buoy cases.

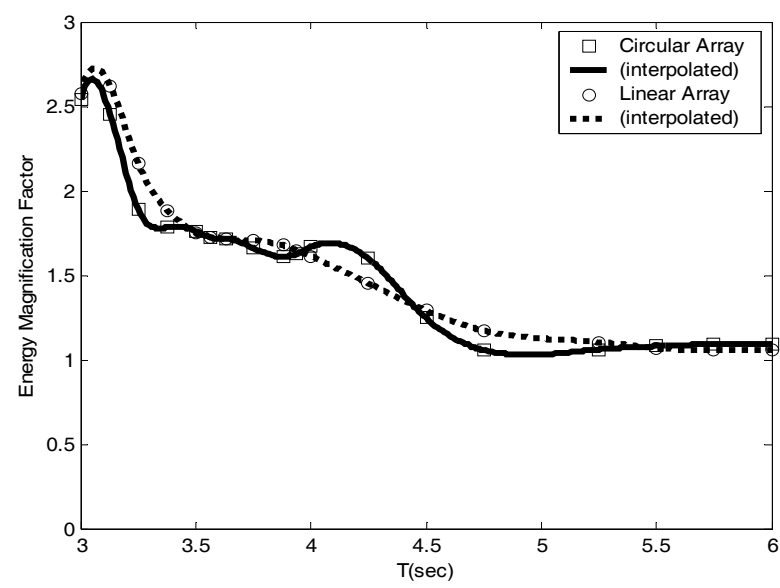

Fig. 6 Comparison between array types for energy magnification factor measured at buoy positions.

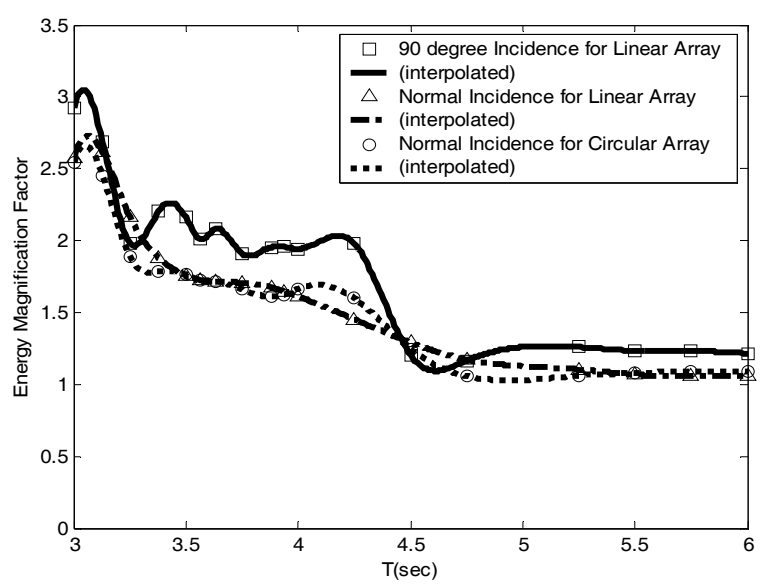

Fig. 8 Comparison of energy magnification factors between 90 degree, 0 degree incidences of linear array and 0 degree incidence of a circular array. 


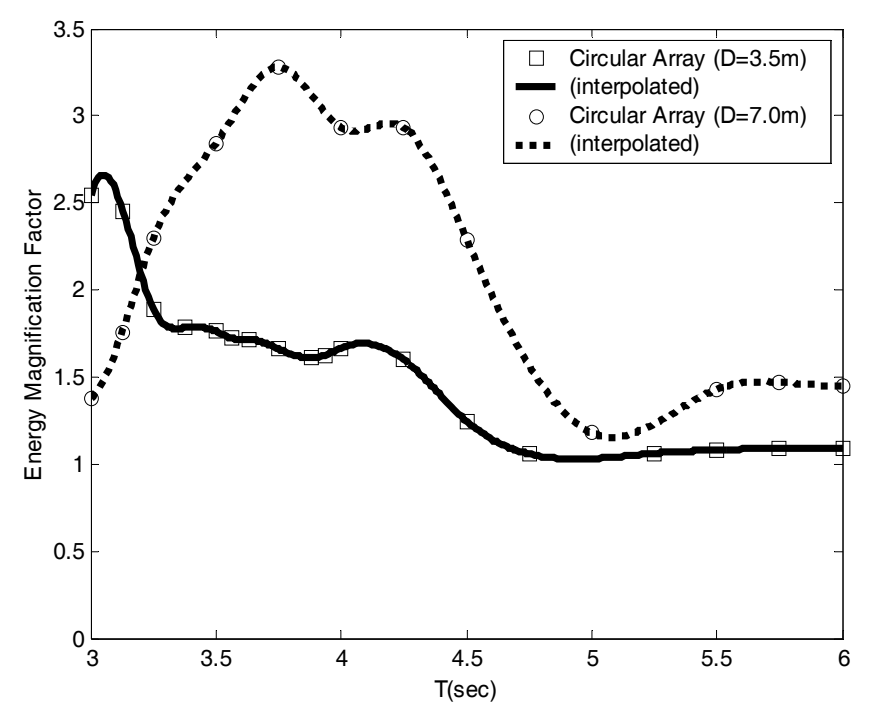

Fig. 9 Effect of buoy diameter on energy magnification factor for a circular array.

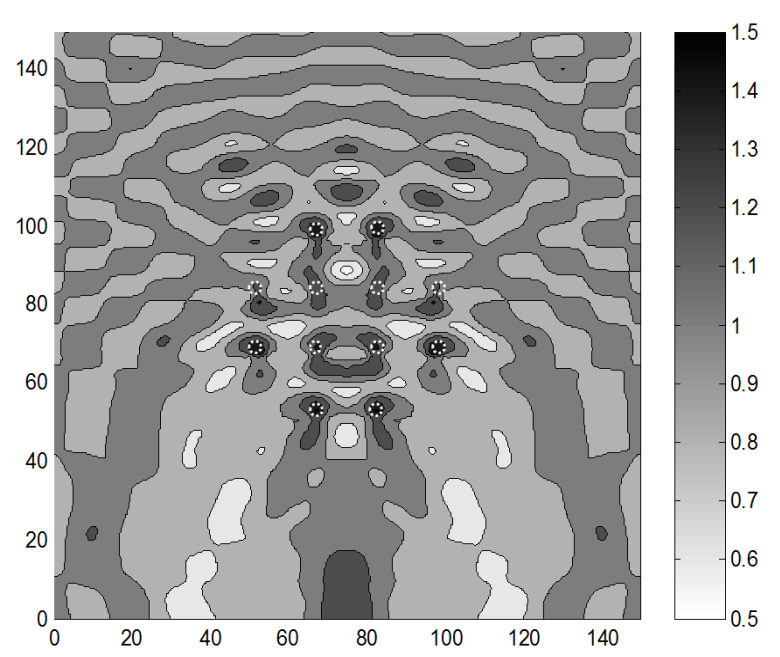

(a) Circular array of normal incidence.

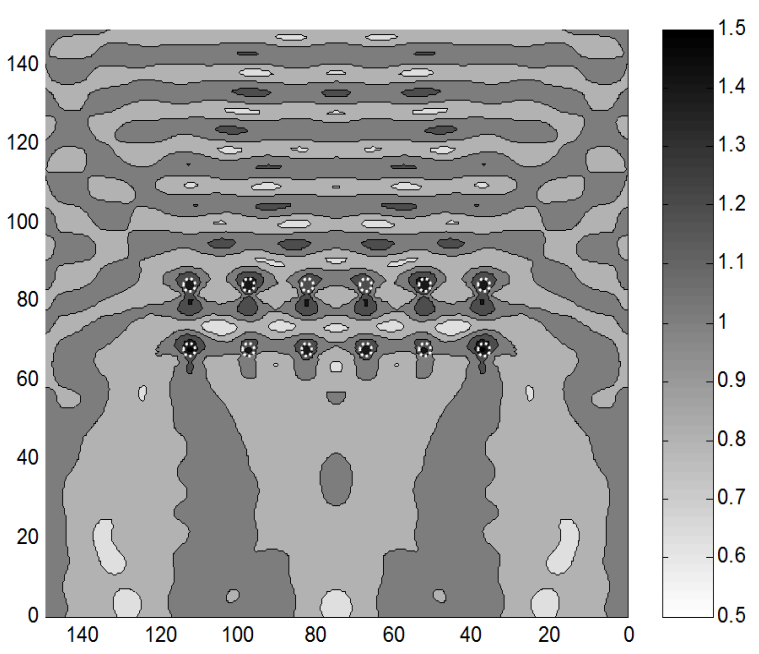

(b) Linear array of normal incidence.

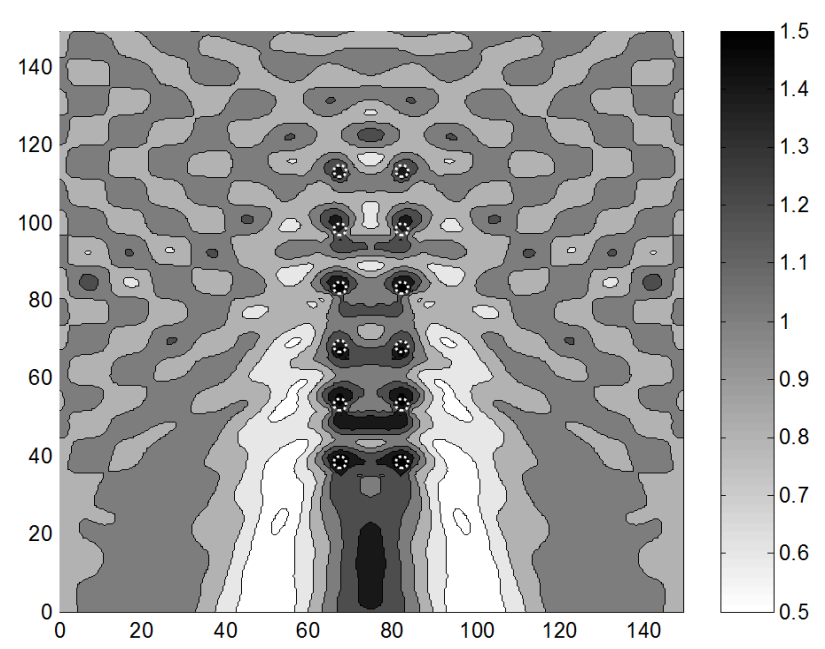

(c) Linear array of 90 degree incidence.

Fig. 10 Wave height distributions of $\mathrm{T}=3.5 \mathrm{sec}$ for floating buoy case: (a) circular array,

(b) linear array of normal incidence, and (c) linear array of 90 degree incidence. 


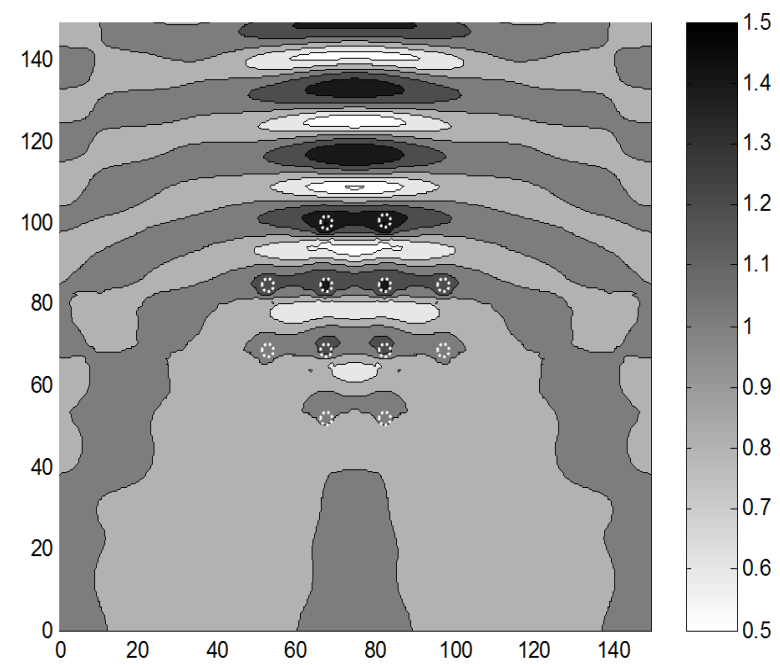

(a) Circular array of normal incidence.

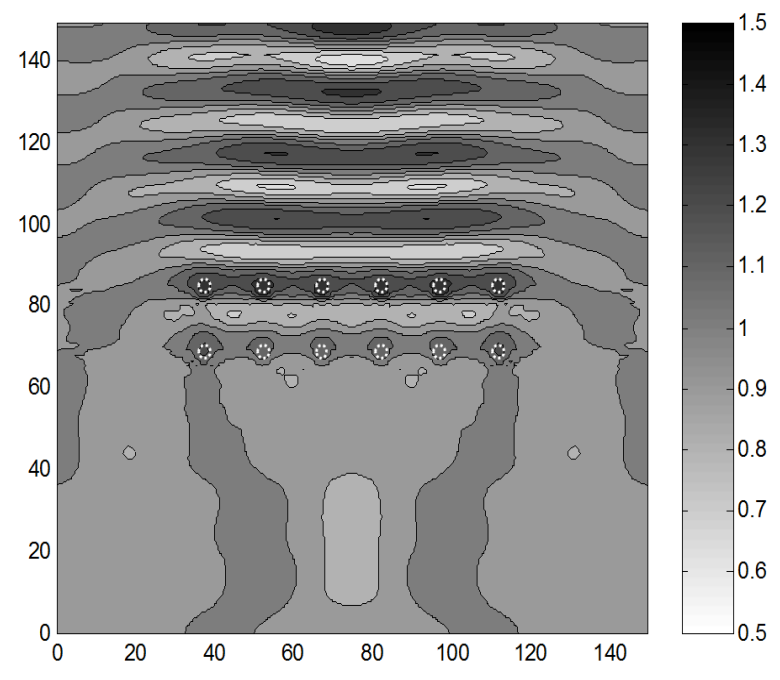

(b) Linear array of normal incidence.

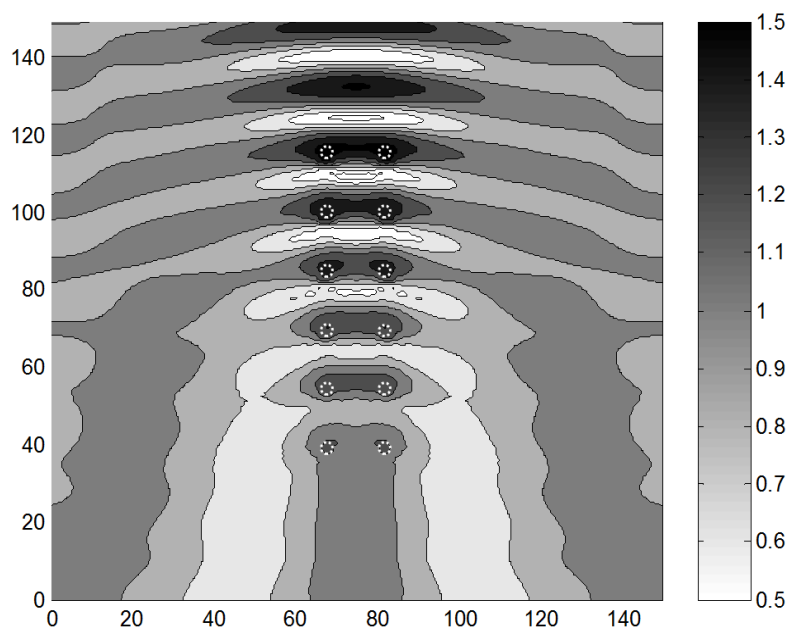

(c) Linear array of 90 degree incidence.

Fig. 11Wave height distributions of $T=4.5 \mathrm{sec}$. for floating buoy case: (a) circular array, (b) linear array of normal incidence, and (c) linear array of 90 degree incidence.

\section{CONCLUSIONS}

From the wave energy feasibility studyon the eastern coast of Korea, choosing a resonant buoy type and further designing its optimal array are required reach a commercial level. To a study the array effect of buoyenergy farms, the present plane wave model is applied to buoy farm configurations with circular and linear arrays, and the numerical results show that the floating-buoy-type energy farm appears to be sufficiently feasible intrappingmore energy compared with afixed cylinder duck array. Therefore, the selection of an optimal buoy array might be important for enhancing the electrical efficiency of buoytype energy farms. The peak periods can be modulated by changing the spacing between adjacent buoys and the floating buoy diameter.

The conventional mild-slope equation was modified by the wide-spacing approximation for the present study to simulate waves scattering against multiple floating devices of a wave energy farm. The modification was done by inclusion of a scattering term given in terms of the solutions for a single device. It is shown that the present method provides satisfactory performance with high computational efficiency as a method applicable to a wide class of wave-structure interaction problems. 


\section{ACKNOWLEDGEMENTS}

This work was conducted through the New and Renewable Energy R\&D Program (Grant KETEP 2010-302007 0080) entitled ‘Wave Energy Farm utilizing by Resonance Power Buoy' funded by the Korea Institute of Energy Technology Evaluation and Planning.

\section{REFERENCES}

Berkhoff, J.C.W., 1972. Computation of combined refraction diffraction. Proceedings of the 13th International Conference on Coastal Engineering. pp.471-490.

Berteaux, H.O., 1976. Buoy engineering. Wiley-Inter Science Publication.

Copeland, G.J.M., 1985. A practical alternative to the mild slope wave equation. Coastal Engineering, 9(2), pp.125-149.

European Thematic Network on Wave Energy (ETNWE), 2003. Results from the work of the European thematic network on wave energy. ERK5-CT-1999-20001, 2000-2003.

Evans, D.V., 1976. A theory for wave-power absorption by oscillating bodies. Journal of Fluid Mechanics, 77(1), pp.1-25.

Kweon, H.M., Kim, I.H. and Lee, J.L., 2011. Rip current control behind steel-typed multiple breakwaters. Journal of Coastal Research, SI(64), pp.1779-1783.

Koutandos, E., Prinos, P. and Gironella, X., 2005. Floating breakwaters under regular and irregular wave forcing: reflection and transmission characteristics. Journal of Hydraulic Research, 43(2), pp.174-188.

Lee, J.L. and Lee, K.J., 2001. Effect of a surface-piercing vertical thin breakwater to harbor tranquility. The First Asian and Pacific Coastal Engineering Conference. pp.186-195.

Lee, J.L. and Lee, D.Y., 2003. Modeling of wave scattering by vertical barriers. Proceedings of 13th International Offshore and Polar Engineering Conference, ISOPE. Honolulu, Hawaii, USA 25-30 May 2003, 3, pp.773-780.

Lee, J.L., Lee, J.Y. and Kim, I.H., 2011. Managing effect of hot spot shoreline behind a power buoy energy farm. Journal of Coastal Research, SI(61), pp.309-316.

Madsen, P.A. and Larsen, J., 1987. An efficient finite-difference approach to the mild-slope equation. Coastal Engineering, 11(4), pp.329-351.

Mavrakos, S.A. and McIver, P., 1997. Comparison of methods for computing hydrodynamic characteristics of arrays of wave power devices. Applied Ocean Research, 19(5-6), pp.283-291.

McIver, P. and Evans, D.V., 1984. Approximation of wave forces on cylinder arrays. Applied Ocean Research, 6(2), pp.101-107.

Ohyama, T. and Tsuchida, M., 1997. Expanded mild-slope equations for the analysis of wave-induced ship motion in a harbor. Coastal Engineering, 30(1-2), pp.77-103.

Oregon Sea Grant Report, 2004. Conversion of wave characteristics to actual electric energy/power potentials.

Radder, A.C., 1979. On the parabolic equation method for water wave propagation. Journal of Fluid Mechanics, 95(1), pp.159-176.

Sawaragi, T., Aoki, S. and Hamamoto, S., 1989. Analysis of hydrodynamic forces due to waves acting on a ship in a harbor of arbitrary geometry. Proceedings of the 8th International Conference on Offshore Mechenics and Arctic Engineering, ASME. pp.117-123.

Simon, M.J., 1982. Multiple scattering in arrays of axisymmetric wave energy devices. Part 1: a matrix method using a plane-wave approximation. Journal of Fluid Mechanics, 120, pp.1-25.

Smith, R. and Sprinks, T., 1975. Scattering of surface waves by a conical islands. Journal of Fluid Mechanics, 72(2), pp. 373-384.

Thorpe, T.W., 1999. A brief review of wave energy. ETSU Report R-122, prepared for the United Kingdom Department of Trade and Industry.

Ursell, F. and Dean, W.R., 1947. The Effect of a fixed vertical barrier on surface waves in deep water. Proceedings of the Cambridge Philosopical Society. 43(30), p.374.

Williams, A.N. and Li, W., 2000. Water wave interactions with an array of bottom mounted surface-piercing porous cylinders. Ocean Engineering, 27(8), pp.841-866. 